\title{
Ophiopogon japonicus strains from different cultivation regions exhibit markedly different properties on cytotoxicity, pregnane $X$ receptor activation and cytochrome P450 3A4 induction
}

\author{
LE-LE GE ${ }^{1}$, LIAN-DI KAN ${ }^{1}$, ZHENG-BING ZHUGE $^{1}, \mathrm{KE} \mathrm{MA}^{1}$ and SHU-QING CHEN ${ }^{2}$ \\ ${ }^{1}$ Department of Pharmacy, Sir Run Run Shaw Hospital, College of Medicine, Zhejiang University, Hangzhou, \\ Zhejiang 310016; ${ }^{2}$ Department of Pharmaceutical Analysis and Drug Metabolism, College of \\ Pharmaceutical Science, Zhejiang University, Hangzhou, Zhejiang 310058, P.R. China
}

Received February 2, 2015; Accepted March 6, 2015

DOI: 10.3892/br.2015.443

\begin{abstract}
Maidong, known as Ophiopogon japonicus, is one of the two basic ingredients of Shenmai injection, which is a widely used herbal preparation in traditional Chinese medicine (TCM) for the treatment of atherosclerotic coronary heart disease and viral myocarditis. Previously, the ethanol extract of Maidong activated the pregnane $X$ receptor (PXR) signaling pathway and induced the cytochrome P450 3A4 (CYP3A4) reporter gene and raised the concern of herb-drug interactions (HDIs) when Maidong was used in combination with prescribed drugs metabolized by CYP3A4. Therefore, the present study further investigated and compared the differences of the ethanol and aqueous extracts (ee- and ae-, respectively) of two Maidong strains, known as Zhe Maidong (ZM) and Chuan Maidong (CM). Cytotoxicity, PXR activation and CYP3A4 induction by the 3-(4,5)-dimethylthiahiazo-(-z-y1)-3,5-diphenytetrazoliumromide assay, reporter gene assay and reverse transcription-quantitative polymerase chain reaction
\end{abstract}

Correspondence to: Dr Ke Ma, Department of Pharmacy, Sir Run Run Shaw Hospital, College of Medicine, Zhejiang University, 3 Qinchun East Road, Hangzhou, Zhejiang 310016, P.R. China

E-mail: srrshmake@126.com

Dr Shu-Qing Chen, Department of Pharmaceutical Analysis and Drug Metabolism, College of Pharmaceutical Science, Zhejiang University, 866 Yuhangtang Road, Hangzhou, Zhejiang 310058, P.R. China

E-mail: chenshuqing@zju.edu.cn

Abbreviations: ee, ethanol extract; ae, aqueous extract; ZM, Zhe Maidong; CM, Chuan Maidong; PXR, pregnane X receptor; CYP3A4, cytochrome $\mathrm{P} 450$ 3A4; TCM, traditional Chinese medicine; HDIs, herb-drug interactions; DMSO, dimethyl sulfoxide; FBS, fetal bovine serum; MTT, 3-(4,5)-dimethylthiahiazo-(-z-y1)-3,5-diphenytetrazoliumromide; RIF, rifampicin; RT-qPCR, reverse transcription-quantitative polymerase chain reaction

Key words: Ophiopogon japonicus, pregnane X receptor, cytochrome $\mathrm{P} 450$ 3A4, herb-drug interactions, traditional Chinese medicine analysis were examined. The observations showed that ee-ZM demonstrated a significantly higher cytotoxicity, a relatively weaker PXR activation capability and a markedly stronger CYP3A4-inducing capacity than ee-CM. Compared to ae-CM, ae-ZM exhibited only a slight or no difference on cytotoxicity and CYP3A4 induction, while a significant lower level of PXR activation was apparent. Collectively, Maidong from different producing areas possess different properties upon cytotoxicity and the drug-metabolizing enzyme inducing effect, and attention should be paid to the selection of Maidong strains from different planting regions into TCM preparations for reducing potential adverse reactions and HDIs.

\section{Introduction}

Herbal medicines are gaining increasing popularity worldwide. There are reportedly $>11,000$ species of herbal plants worldwide and $~ 500$ species are commonly used in Asian and other countries (1). However, herb-drug interactions (HDIs) have gained increasing concerns following the increasingly frequent combination use of herbal medicines and pharmaceutical drugs. Concurrent use of herbs could mimic, magnify or oppose the effect of drugs (2). For example, prothrombin time was prolonged, and consequently, bleeding may occur when warfarin is combined with ginkgo (Ginkgo biloba) (3), dong quai (Angelica sinensis) (4) or danshen (Salvia miltiorrhiza) (5-7). St. John's wort (Hypericum perfo ratum) can oppose the effect of theophylline (8), digoxin (9) and cyclosporine (10) through decreasing their concentrations in serum.

Cytochrome P450 (CYP), an important phase I drug-metabolizing enzyme system, is responsible for the metabolism of a wide range of xenobiotics, including therapeutic drugs and certain important endogenous substances, and cytochrome P450 3A4 (CYP3A4), the major isoenzyme of the hepatic CYPs in humans, is involved in the metabolism of approximately half the drugs currently in use (11). The pregnane $\mathrm{X}$ receptor (PXR), also known as NR1I2, SXR or PAR and an important component of the body's adaptive defense mechanism against toxic substances including xenobiotics, has been identified and verified as a critical regulator of $C Y P 3 A 4$ gene expression $(12,13)$. PXR can be ligand-activated by a vast 
variety of endogenous and exogenous chemicals, such as steroids (14), therapeutic drugs (15), herbal medicines (16) and environmental pollutants (17), and the activated PXR, along with other cofactors, finally binds to the promoter region of CYP3A4 and subsequently upregulates CYP3A4 transcription. The PXR-CYP3A4 pathway has been clarified as a common mechanism mediating the HDIs. For instance, St. John's wort was found to induce $C Y P 3 A 4$ expression through activation of the PXR (18) and cryptotanshinone and tanshinone IIA, the major bioactive components from Danshen herb, were identified as efficacious PXR agonists and induced CYP3A4 expression via the PXR-CYP3A4 pathway (19).

Maidong is one of the two basic ingredients of Shenmai injection, one of the most widely used herbal medicines in traditional Chinese medicine (TCM) and frequently used to treat atherosclerotic coronary heart disease and viral myocarditis or co-administered with other prescribed medicines in certain circumstances as an organ protector $(20,21)$. Recently, our previous study screened and identified that the ethanol extract of Maidong activated the PXR-CYP3A4 pathway using a reporter gene system, suggesting the possible HDIs when Maidong or Maidong-related preparations are co-used with prescribed drugs metabolized by CYP3A4 (22).

The present study further investigated and compared effects of the extracts of Zhe Maidong (ZM) and Chuan Maidong (CM), two Maidong strains cultivated in the Zhejiang and Sichuan provinces, which are the major planting regions in China, respectively, on the cytotoxicity and capacity of PXR activation and CYP3A4 induction using an in vitro cell-based reporter gene system and reverse transcription-quantitative polymerase chain reaction (RT-qPCR) analysis. In addition, a comparison was also made on the cytotoxicity between the extracts of ZM and CM quantitatively by the 3-(4,5)-dimethylthiahiazo-(-z-y1)-3,5-diphenytetrazoliumromide (MTT) assay. The results revealed that the extracts of ZM and CM have significantly different cytotoxicity, PXR activation capability and $C Y P 3 A 4$ induction capacity, suggesting that herb strains from different cultivation regions should also be evaluated and considered in preparing TCM prescriptions to reduce or avoid potential adverse reactions and HDIs.

\section{Materials and methods}

Chemicals and reagents. Dimethyl sulfoxide (DMSO), MTT and non-essential amino acids were obtained from Sigma-Aldrich (St. Louis, MO, USA). The plasmids, pcDNA3.1-hPXR, pGL3-PXRE and pRL-TK, were constructed as previously described (19). Mega Tran 1.0 transfection reagent was purchased from OriGene (Rockville, MD, USA). The dual-luciferase reporter assay system was provided by Promega Corporation (Madison, WI, USA). The E.Z.N.A. ${ }^{\text {TM }}$ Endo-free Plasmid mini kit was the product of Omega Bio-Tek, Inc. (Norcross, GA, USA). SYBR ${ }^{\circledR}$ Premix Ex Taq ${ }^{\mathrm{TM}}$ was purchased from Takara Bio, Inc. (Shiga, Japan). The primers used in qPCR were synthesized by Invitrogen Life Technologies (Shanghai, China). Rifampicin (RIF) was obtained from Sangon Biotech Co. Ltd. (Shanghai, China). CM and ZM were obtained from Huqingyutang Pharmaceutical Co., Ltd. (Zhejiang, China). All the other chemicals used were of analytical grade.
Cell lines and culture.Human hepatic carcinoma cell line HepG2 and colon adenocarcinoma cell line LS174T were obtained from the Institute of Biochemistry and Cell Biology (Shanghai, China). The cell lines were cultured in Dulbecco's modified Eagle's medium (Invitrogen Life Technologies, Carlsbad, CA, USA) supplemented with $10 \%$ charcoal/dextran-treated fetal bovine serum (HyClone, Logan, UT, USA), 1\% non-essential amino acids, $1 \%(\mathrm{v} / \mathrm{v})$ penicillin and streptomycin (Corning Life Sciences, Oneonta, NY, USA). Cells were maintained routinely at $37^{\circ} \mathrm{C}$ in a $5 \% \mathrm{CO}_{2}$ humidified atmosphere.

Ethanol and aqueous extracts of Maidong. Ethanol and aqueous extracts (ee- and ae-, respectively) of Maidong from two cultivation regions (ee-ZM, ee-CM, ae-ZM and ae-CM) were prepared with the heat reflux extraction method. To prepare the aqueous extracts of Maidong, the raw herbs were ground and soaked in an 8-fold volume of distilled water for $30 \mathrm{~min}$ and extracted by heat reflux extraction for $30 \mathrm{~min}$ in a boiling water bath. The herbs were heat reflux extracted three times, combined together and filtered, respectively. The filtrates were concentrated by a rotary evaporator and further dried by freeze drying, and the yield of dry extracts was calculated. Ethanol extracts were prepared in the same way as the aqueous extracts, except that the solvent was changed to $80 \%$ ethanol and the water bath temperature to $80^{\circ} \mathrm{C}$. The dry extract yield was calculated in the same way.

MTT assay. HepG2 cells were seeded in 96-well plates at a density of 5,000 cells/well, allowed to attach overnight and were treated with Maidong extracts at indicated concentrations for $48 \mathrm{~h}$. Medium containing herb extracts were renewed every $24 \mathrm{~h}$. After treatment, $20 \mu \mathrm{l}$ of the $5 \mathrm{mg} / \mathrm{ml}$ MTT was added to each well and incubated for $4 \mathrm{~h}$ at $37^{\circ} \mathrm{C}$. The supernatant was removed carefully and $150 \mu \mathrm{l}$ of DMSO was added to each well. Ten minutes after incubation at $37^{\circ} \mathrm{C}$, the absorbance value of each well was read at $490 \mathrm{~nm}$ using an ELISA plate reader instrument (model 680; Bio-Rad, Tokyo, Japan).

Reporter gene assay. Reporter gene assays were performed as described previously (19) with a slight modification. Briefly, plasmid DNA was prepared by the E.Z.N.A. ${ }^{\text {TM }}$ Endo-free Plasmid Mini kit. HepG2 cells were seeded in 24-well plates at a density of $2.5 \times 10^{4}$ cells/well and allowed to attach overnight, and were transiently co-transfected with pcDNA3.1-hPXR, pGL3-PXRE and pRL-TK with the aid of Mega Tran 1.0 transfection reagent. Twenty-four hours later, the transfected cells were treated with medium containing aqueous extracts, ethanol extracts, $10 \mu \mathrm{M}$ RIF (positive control) or $0.1 \%$ DMSO (blank control) for an additional $48 \mathrm{~h}$. Medium containing drugs were renewed every $24 \mathrm{~h}$. Following the treatment, the cells were rinsed twice with cold phosphate-buffered saline and were lysed. Firefly luciferase activity was determined and normalized against Renilla luciferase activity. Fold induction was calculated as the normalized reporter activity of a test drug divided by the solvent control (DMSO). Data are shown as the percentage of fold induction of a test extract relative to $10 \mu \mathrm{M}$ RIF and represent the mean of three independent experiments. The test extracts were categorized into three groups: No response or negligible $(<30 \%)$, weak to moderate $(30-70 \%)$ and strong $(>70 \%)$, as previously described $(23,24)$. 
$R T-q P C R$. LS174T cells were seeded in 24-well plates at a density of $5 \times 10^{4}$ cells/well and allowed to attach overnight. Cells were subsequently treated with Maidong extracts at indicated concentrations for $72 \mathrm{~h}$ and $0.1 \%$ DMSO and $10 \mu \mathrm{M}$ RIF were used as the blank and positive control, respectively. Medium containing drugs were renewed every $24 \mathrm{~h}$. After treatment, total RNA was extracted from monolayers of LS174T cells using the Ultrapure RNA kit (CWBio, Beijing, China) according to the manufacturer's instructions. The RNA quality and quantity of samples was assessed by the $260 / 280 \mathrm{~nm}$ absorbance ratios and the $260 \mathrm{~nm}$ absorbance, respectively, using a NanoDrop spectrophotometer (Thermo Fisher Scientific Inc., Beijing, China). Total RNA $(2 \mu \mathrm{g})$ from each sample was used to synthesize cDNA using the Hifi-MMLV cDNA kit (CWBio) according to the manufacturer's instructions. All the PCR reactions were carried out using $\mathrm{SYBR}^{\circledR}$ Premix Ex Taq ${ }^{\mathrm{TM}}$ on an ABI PRISM 7500 qPCR system. Oligonucleotide primers for $C Y P 3 A 4$ and $G A P D H$ were 5'-GTGGGGCTTTTATGATGGTCA-3', 5'-ACATCTCCA TACTGGGCAATGA-3'; and 5'-CTTAGCACCCCTGGC CAAG-3', 5'-GATGTTCTGGAGAGCCCCG-3', respectively. The experiments were run in triplicate as follows: Initial denaturation step of $95^{\circ} \mathrm{C}$ for $30 \mathrm{sec}$, followed by 40 cycles of $95^{\circ} \mathrm{C}$ for $5 \mathrm{sec}$ and $60^{\circ} \mathrm{C}$ for $34 \mathrm{sec}$. Quantitation of CYP3A4 mRNA in treated samples versus vehicle control samples was calculated by correcting for GAPDH in each sample $(\triangle \mathrm{Ct})$ using the equation: Fold induction $=2^{-\Delta \Delta C \mathrm{t}}$.

Statistical analysis. All the statistical analyses were carried out using the SPSS 19.0 statistical software package (SPSS Inc., Chicago, IL, USA). Data are presented as mean \pm standard deviation from one of three independent experiments. One-way analysis of variance was used for the statistical comparisons. $\mathrm{P}<0.05$ was considered to indicate a statistically significant difference.

\section{Results}

Verification of PXR and CYP3A4 mRNA expression of HepG2 and LS174T cells. In the present study, HepG2 cells with an extremely low level of $P X R$ and $C Y P 3 A 4$ expression and LS-174T cells with a high level of $P X R$ and $C Y P 3 A 4$ expression were used as cell models in the reporter gene assay and RT-qPCR analysis to investigate PXR activation and CYP3A4 induction, respectively. First, RT-qPCR analysis was performed to compare and verify the PXR and CYP3A4 mRNA expression of these two cell lines. The data showed that the $P X R$ mRNA expression level was extremely low (mean value, $6.06 \times 10^{-6}$ vs. 1 ) and the CYP3A4 mRNA level was also extremely low (mean value, 0.074 vs. 1) in HepG2 cells compared to LS-174T cells (Fig. 1). The results demonstrate that the HepG2 and LS-174T cells were qualified for further analysis.

Cytotoxicity of CM and ZM extracts. To compare the cytotoxicity of the $\mathrm{CM}$ and $\mathrm{ZM}$ extracts, the relative viability of HepG2 cells treated with ae-CM, ee-CM, ae-ZM and ee-ZM at various concentrations for $48 \mathrm{~h}$ was evaluated by the MTT assay. As shown in Fig. 2, ee-ZM inhibited cell viability in a significant dose-dependent manner and showed a significant

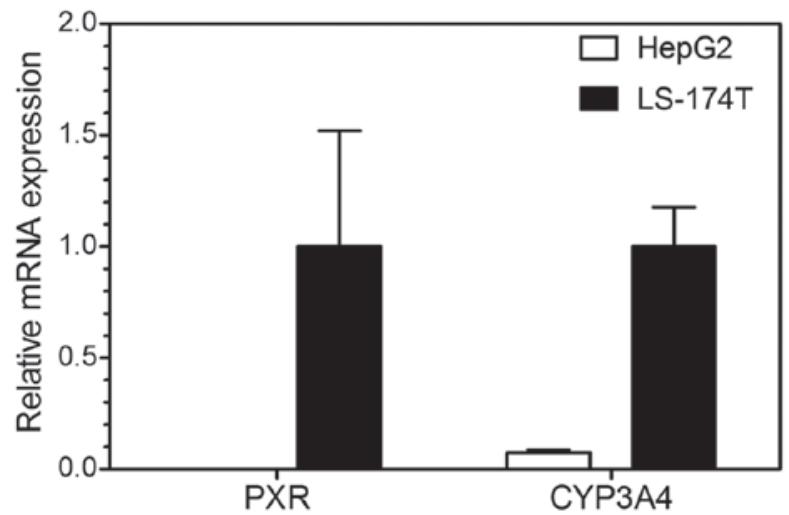

Figure 1. Verification of mRNA expression of the pregnane X receptor (PXR) and cytochrome P450 3A4 (CYP3A4) in human hepatocellular liver carcinoma (HepG2) and LS-174T cells. The relative mRNA level of PXR and CYP3A4 in HepG2 and LS-174T cells was detected by reverse transcription-quantitative polymerase chain reaction. GAPDH was used as internal control.

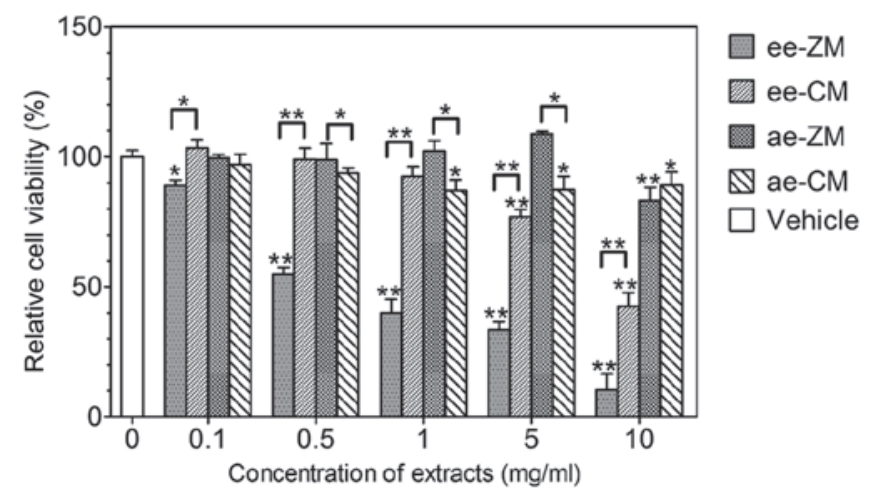

Figure 2. Cytotoxicity of CM and ZM extracts. The effect of CM and ZM extracts on cell viability was determined by the 3-(4,5)-dimethylthiahiazo-(-z-y1)-3,5-diphenytetrazoliumromide assay. When not indicated by fold lines, ${ }^{*}$ and ${ }^{* *}$ represent significant differences from the vehicle (dimethyl sulfoxide) treated group; ${ }^{*} \mathrm{P}<0.05,{ }^{* *} \mathrm{P}<0.001$. CM, Chuan Maidong; ZM, Zhe Maidong; ee, ethanol extract; ae, aqueous extract.

inhibition effect even at the concentration of $0.1 \mathrm{mg} / \mathrm{ml}$, while ee-CM had no clear effect on cell viability when the concentration was not $>1 \mathrm{mg} / \mathrm{ml}$. Furthermore, at every concentration level used, ee-ZM demonstrated higher toxicity to HepG2 cells than ee-CM. As for the aqueous extracts of two Maidong strains, overall they showed a much lower cytotoxicity than their ethanol extract counterparts and ae-CM only showed a slight or moderately more cytotoxicity than ee-CM (Fig. 2). These data indicate that ee-ZM possesses a markedly higher cytotoxicity than ee-CM, while ae-ZM and ae-CM have no evident difference on cytotoxicity.

Activation of human PXR by extracts of CM and ZM. The reporter gene assay was carried out to evaluate and compare the activation of human PXR by the ethanol and aqueous extracts of $\mathrm{CM}$ and $\mathrm{ZM}$. As presented in Fig. 3, overall the extracts of ZM and CM activate human PXR in a dose-dependent manner at the range of $5 \mathrm{mg} / \mathrm{ml}$. The ee-ZM and ee-CM showed no response or negligible effect $(<30 \%)$ only at the concentration of $0.1 \mathrm{mg} / \mathrm{ml}$. The ee-CM had a weak to moderate effect, while ee-ZM also had a negligible effect when the dose was 


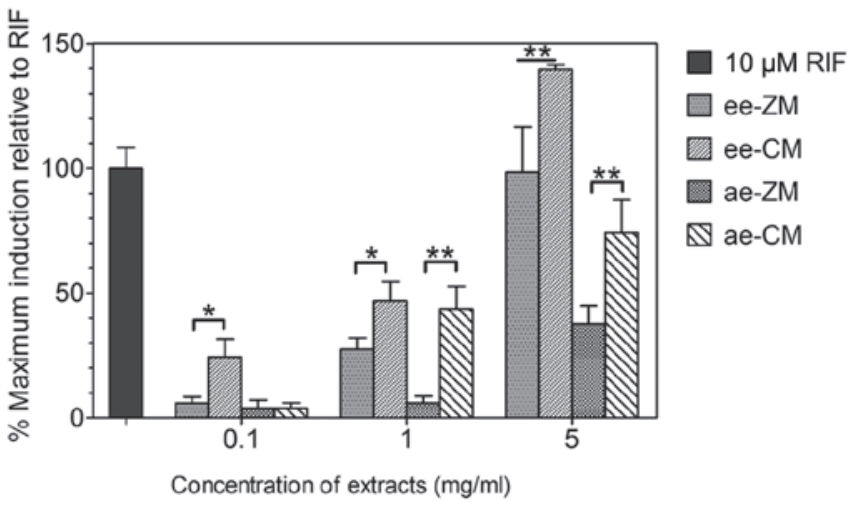

Figure 3. Activation of human pregnane $\mathrm{X}$ receptor (PXR) by $\mathrm{CM}$ and $\mathrm{ZM}$ extracts. The activation effects on human PXR by extracts of CM and ZM were evaluated by the reporter gene assay. Rifampicin (RIF; $10 \mu \mathrm{M}$ ) was used as a positive control. Following treatment, firefly luciferase activity was determined and normalized against Renilla luciferase activity. ${ }^{*} \mathrm{P}<0.05$, ${ }^{* *} \mathrm{P}<0.001$. CM, Chuan Maidong; ZM, Zhe Maidong; ee, ethanol extract; ae, aqueous extract.

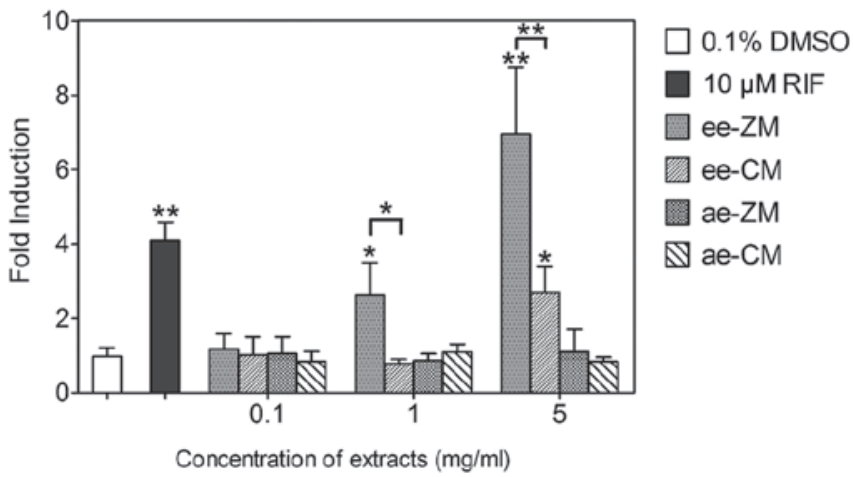

Figure 4. Induction of cytochrome P450 3A4 (CYP3A4) mRNA by CM and $\mathrm{ZM}$ extracts. The induction of $C Y P 3 A 4$ mRNA by extracts of $\mathrm{CM}$ and $\mathrm{ZM}$ was investigated and compared by RT-qPCR analysis. Dimethyl sulfoxide (DMSO; 0.1\%) and $10 \mu \mathrm{M}$ rifampicin (RIF) were used as the blank and positive control, respectively. When not indicated by fold lines, ${ }^{*}$ and ${ }^{* *}$ represent significant differences from the vehicle (DMSO) treated group; ${ }^{*} \mathrm{P}<0.05$, ${ }^{* *} \mathrm{P}<0.001$. CM, Chuan Maidong; ZM, Zhe Maidong; ee, ethanol extract; ae, aqueous extract.

$<1 \mathrm{mg} / \mathrm{ml}$. Furthermore, ee-ZM and ee-CM demonstrated a strong activation effect on human PXR at the dose of $5 \mathrm{mg} / \mathrm{ml}$. The ee-CM exhibited a significantly higher percentage of maximum induction relative to RIF compared to ee-ZM at each concentration (Fig. 3). The ae-ZM and ae-CM showed a similar pattern to their ethanol counterparts. The ae-CM activated human PXR much stronger than the ee-ZM in the reporter gene assay (Fig. 3).

Induction of CYP3A4 mRNA by CM and ZM extracts. To further investigate and compare the induction effect on CYP3A4 mRNA by the ethanol and aqueous extracts of CM and ZM, RT-qPCR analysis was performed. DMSO (0.1\%; vehicle) and $10 \mu \mathrm{M}$ RIF were used as a blank and positive control, respectively. Compared to the vehicle-treated group, only the 1 and $5 \mathrm{mg} / \mathrm{ml}$ ee-ZM and $5 \mathrm{mg} / \mathrm{ml}$ ee-CM groups induced $C Y P 3 A 4 \mathrm{mRNA}$ expression significantly. Additionally, ee-ZM demonstrated a markedly higher induction of CYP3A4
mRNA expression than ee-CM at the concentration of 1 and $5 \mathrm{mg} / \mathrm{ml}$ (Fig. 4). The ae-ZM and ae-CM did not exhibit an evident induction effect on $C Y P 3 A 4$ mRNA expression at all the used concentrations (Fig. 4).

\section{Discussion}

HDIs have been evident for a long time and increasing extracts or single components of herbs have been identified as inducers of drug-metabolizing enzymes that are attributable to various HDIs. Shenmai injection, an injection made of Renshen (Panax ginseng) and Maidong (Ophiopogon japonicus), is commonly used to treat atherosclerotic coronary heart disease and viral myocarditis (20). Currently, it has been also widely applied in clinical practice as an organ protector (21). Generally, Shenmai injection is considered effective and safe for clinical application. However, it was reported that Shenmai injection could inhibit the activities of hepatic CYP3A1/2 and CYP2C6 (25) and differentially affect CYP3A4-mediated 1'-hydroxylation and 4-hydroxylation of midazolam (20), suggesting potential HDIs could occur when it is co-used with other therapeutic drugs. Our previous study screened the ethanol extracts of 28 commonly used herb medicines and 34 bioactive components from these herb medicines for their capability to activate the PXR-CYP3A4 pathway. The data demonstrated that 22 herbal medicine ethanol extracts and 8 bioactive compounds activated PXR and induced the CYP3A4 reporter gene. Among them, the ethanol extract of Maidong strongly activated PXR (22).

In China, Zhejiang, a southeast coastal province, and Sichuan, an inland province, are the major cultivation regions of Maidong. In addition to growth area, ZM and CM are also different in respects of planting conditions, growth period and processing and it is well-documented that the environmental and growing conditions significantly influence the quantity and quality of herbs grown in different geographic regions. Lin et al (26) reported that homoisoflavonoids, one of the main bioactive components of Maidong, showed a similar chemical profile but distinctive combination and ratio in ZM (also known as Hang Maidong in the study as Hangzhou is the provincial capital of Zhejiang) and CM. However, to the best of our knowledge, whether ZM and CM possess different properties on PXR activation and CYP3A4 induction has not been reported yet.

The present study further investigated and compared the effects of the ethanol and aqueous extracts of two Maidong strains from different cultivation regions, ZM and CM, on cytotoxicity, PXR activation and CYP3A4 induction, respectively. The ee-ZM activated PXR and induced the $C Y P 3 A 4$ reporter gene using the HepG2-based reporter gene system described previously (19) and this result was further supported by the data of RT-qPCR analysis in the LS-174T cells, which is in line with and further verified our previous observations (22), indicating that ee-ZM contains bioactive compounds that activated the PXR-CP3A4 pathway. Of note, ee-CM showed a markedly stronger activation effect on PXR than ee-ZM, while there was a much weaker effect on $C Y P 3 A 4$ induction. This phenomenon suggests that ee-CM activates $P X R$ and may mainly regulate other PXR-target genes expression, such as other CYP isoenzymes since a large number of genes involved in the oxidation 
(phase I), conjugation (phase II) and transport (phase III) of xenobiotics are regulated by PXR in the liver (13). Therefore, ee-ZM is more likely to cause $C Y P 3 A 4$-mediated HDIs than ee-CM. As for the aqueous extracts of Maidong, the ae-ZM and ae-CM exhibited no significant induction of $C Y P 3 A 4$ expression, although the ae-CM appears to be a relatively stronger activator of PXR. Additionally, we also demonstrated that ee-ZM has a much higher cytotoxicity than ee-CM, and ae-ZM and ae-CM exhibited only a slight cytotoxicity at high concentrations.

In conclusion, the present study demonstrated that ZM possesses a significantly higher cytotoxicity, stronger induction of CYP3A4 expression and relatively weaker activation on PXR than CM, suggesting more potential adverse reactions and $C Y P 3 A 4$-mediated HDIs may occur when $\mathrm{ZM}$ is co-administered with other prescribed drugs compared to CM. However, major effector molecules of the PXR signaling pathway activated by ee-CM are required to further elucidate the investigation. The present results suggest that herb strains from different cultivation regions should be evaluated and considered in preparing TCM prescriptions to reduce potential adverse reactions and HDIs.

\section{Acknowledgements}

The authors would like to thank Dr Hui Fan and Mr. Zhenwei Yu for their excellent technical assistance. The present study was supported by the Science and Technology Research Project of Zhejiang Province, China (grant nos. 2011KYA089 and 2011ZA062).

\section{References}

1. Liu YH, Mo SL, Bi HC, Hu BF, Li CG, Wang YT, Huang L, Huang M, Duan W, Liu JP, et al: Regulation of human pregnane $\mathrm{X}$ receptor and its target gene cytochrome P450 3A4 by Chinese herbal compounds and a molecular docking study. Xenobiotica 41: 259-280, 2011.

2. Fugh-Berman A: Herb-drug interactions. Lancet 355: 134-138, 2000.

3. Matthews MK Jr: Association of Ginkgo biloba with intracerebral hemorrhage. Neurology 50: 1933-1934, 1998.

4. Page RL II and Lawrence JD: Potentiation of warfarin by dong quai. Pharmacotherapy 19: 870-876, 1999.

5. Tam LS, Chan TY, Leung WK and Critchley JA: Warfarin interactions with Chinese traditional medicines: Danshen and methyl salicylate medicated oil. Aust N Z J Med 25: 258, 1995.

6. Yu CM, Chan JC and Sanderson JE: Chinese herbs and warfarin potentiation by 'danshen'. J Intern Med 241: 337-339, 1997.

7. Izzat MB, Yim AP and El-Zufari MH: A taste of Chinese medicine! Ann Thorac Surg 66: 941-942, 1998.

8. Nebel A, Schneider BJ, Baker RK and Kroll DJ: Potential metabolic interaction between St. John's wort and theophylline. Ann Pharmacother 33: 502, 1999.

9. Johne A, Brockmöller J, Bauer S, Maurer A, Langheinrich M and Roots I: Pharmacokinetic interaction of digoxin with an herbal extract from St John's wort (Hypericum perforatum). Clin Pharmacol Ther 66: 338-345, 1999.
10. Bauer S, Störmer E, Johne A, Krüger H, Budde K, Neumayer HH, Roots I and Mai I: Alterations in cyclosporin A pharmacokinetics and metabolism during treatment with St John's wort in renal transplant patients. Br J Clin Pharmacol 55: 203-211, 2003.

11. Zhou S, Gao Y, Jiang W, Huang M, Xu A and Paxton JW: Interactions of herbs with cytochrome P450. Drug Metab Rev 35: 35-98, 2003.

12. LeCluyse EL: Pregnane $X$ receptor: Molecular basis for species differences in CYP3A induction by xenobiotics. Chem Biol Interact 134: 283-289, 2001.

13. Kliewer SA, Goodwin B and Willson TM: The nuclear pregnane $\mathrm{X}$ receptor: A key regulator of xenobiotic metabolism. Endocr Rev 23: 687-702, 2002.

14. Ripp SL, Fitzpatrick JL, Peters JM and Prough RA: Induction of CYP3A expression by dehydroepiandrosterone: Involvement of the pregnane X receptor. Drug Metab Dispos 30: 570-575, 2002.

15. Lehmann JM, McKee DD, Watson MA, Willson TM, Moore JT and Kliewer SA: The human orphan nuclear receptor PXR is activated by compounds that regulate CYP3A4 gene expression and cause drug interactions. J Clin Invest 102: 1016-1023, 1998.

16. Mu Y, Zhang J, Zhang S, Zhou HH, Toma D, Ren S, Huang L, Yaramus M, Baum A, Venkataramanan R, et al: Traditional Chinese medicines Wu Wei Zi (Schisandra chinensis Baill) and Gan Cao (Glycyrrhiza uralensis Fisch) activate pregnane X receptor and increase warfarin clearance in rats. J Pharmacol Exp Ther 316: 1369-1377, 2006.

17. Coumoul X, Diry M and Barouki R: PXR-dependent induction of human CYP3A4 gene expression by organochlorine pesticides. Biochem Pharmacol 64: 1513-1519, 2002.

18. Moore LB, Goodwin B, Jones SA, Wisely GB, Serabjit-Singh CJ, Willson TM, Collins JL and Kliewer SA: St. John's wort induces hepatic drug metabolism through activation of the pregnane $\mathrm{X}$ receptor. Proc Natl Acad Sci USA 97: 7500-7502, 2000.

19. Yu C, Ye S, Sun H, Liu Y, Gao L, Shen C, Chen S and Zeng S: PXR-mediated transcriptional activation of CYP3A4 by cryptotanshinone and tanshinone IIA. Chem Biol Interact 177: 58-64, 2009.

20. Zeng C, He F, Xia C, Zhang $\mathrm{H}$ and Xiong Y: Identification of the active components in Shenmai injection that differentially affect Cyp3a4-mediated 1'-hydroxylation and 4-hydroxylation of midazolam. Drug Metab Dispos 41: 785-790, 2013.

21. Lu LY, Zheng GQ and Wang Y: An overview of systematic reviews of shenmai injection for healthcare. Evid Based Complement Alternat Med 2014: 840650, 2014.

22. Yu C, Chai X, Yu L, Chen S and Zeng S: Identification of novel pregnane $\mathrm{X}$ receptor activators from traditional Chinese medicines. J Ethnopharmacol 136: 137-143, 2011.

23. Luo G, Cunningham M, Kim S, Burn T, Lin J, Sinz M, Hamilton G, Rizzo C, Jolley S, Gilbert D, et al: CYP3A4 induction by drugs: Correlation between a pregnane $\mathrm{X}$ receptor reporter gene assay and CYP3A4 expression in human hepatocytes. Drug Metab Dispos 30: 795-804, 2002.

24. Jacobs MN, Nolan GT and Hood SR: Lignans, bacteriocides and organochlorine compounds activate the human pregnane $\mathrm{X}$ receptor (PXR). Toxicol Appl Pharmacol 209: 123-133, 2005.

25. Xia CH, Sun JG, Wang GJ, Shang LL, Zhang XX, Zhang R, Peng Y, Wang XJ, Hao HP, Xie L, et al: Herb-drug interactions: In vivo and in vitro effect of Shenmai injection, a herbal preparation, on the metabolic activities of hepatic cytochrome P450 3A1/2, 2C6, 1A2 and 2E1 in rats. Planta Med 76: 245-250, 2010.

26. Lin Y, Zhu D, Qi J, Qin M and Yu B: Characterization of homoisoflavonoids in different cultivation regions of Ophiopogon japonicus and related antioxidant activity. J Pharm Biomed Anal 52: 757-762, 2010. 